\title{
Primary Prostate Cancer Tissue/hTERT/Survivin mRNA-loaded Autologous Dendritic Cell Vaccine
}

\author{
National Cancer Institute
}

\section{Source}

National Cancer Institute. Primary Prostate Cancer Tissue/hT ERT/SUrvivin mRNA-loaded

Autologous Dendritic Cell Vaccine. NCI Thesaurus. Code C95771.

An autologous dendritic cells (DCs) vaccine targeting prostate cancer with

immunostimulating activity. The autologous DC vaccine is prepared via transfecting DCs

with mRNAs extracted from primary prostate cancer tissue, and mRNAs of human telomerase reverse transcriptase (hTERT) and survivin. Upon administration, this DC vaccine may elicit a potent cytotoxic T-cell (CTL) response against prostate cancer cells, resulting in tumor cell death. Both hTERT and survivin are essential in neoplastic growth, and are considered to be universal tumor antigens. 